\title{
EXTENDED X-RAY ABSORPTION FINE STRUCTURE STUDIES OF Co DOPED ZnS AND ZnSe ALLOYS
}

\author{
K. ŁAWNICZAK-JABŁOŃSKA AND Z. GolACKI \\ Institute of Plysics, Polish Academy of Science \\ Al. Lotników, 02-668 Warszawa, Poland
}

$\mathrm{X}$-ray absorption experiments were performed at the $K$-edge of $\mathrm{Co}$ and $\mathrm{Zn}$ in $\mathrm{Zn}_{1-x} \mathrm{Co}_{x} \mathrm{~S}$ and $\mathrm{Zn}_{1-x} \mathrm{Co}_{x}$ Se compounds with $x=0.25,0.16,0.10$, $0.05,0.00$ in the sulphides samples and $x=0.07,0.02,0.00$ in the selenide ones. Analysis of the extended X-ray absorption fine structure oscillations using phase and amplitudes either from McKale code or from standard samples, gave the distance, number of atoms and the Debye-Waller factors for nearest neighbours. We found that the cation-anion distances $\mathrm{Zn}-\mathrm{S}(\mathrm{Se})$ or Co-S(Se) are systematically shorter in $\mathrm{ZnS}$ than in $\mathrm{ZnSe}$ matrix, the $\mathrm{Zn}$-anion distance is always larger than the Co-anion one without any significant Co content dependence. This allowed us to estimate the covalent radius of Co in the studied matrices to be $0.025 \AA$ smaller than the $\mathrm{Zn}$ covalent radius and stated that covalent radius of Co is independent of the type of surrounding atoms. In addition, the Debye-Waller factors indicated a better ordering in ternary compounds than in the binary standard ones in agreement with rocking curve measurements.

PACS numbers: 61.10.Lx, 87.64.Fb, 78.70.Dm

\section{Introduction}

Great interest of scientists in the technology and properties of ternary alloys is associated with the increasing expectations of the electronic industry for the physical, optical, mechanical and chemical properties of modern materials. The alloying of elements makes it possible, in many cases, to change continuously the properties of materials e.g. the lattice parameters, energy gap, conductivity, magnetic moment, plasticity, corrosion endurance etc. Investigations of the atomic short range order and the electronic structure in the new classes of ternary alloys provides indications for more general rules for mixing of materials. Such studies can also explain reasons for very limited solid solubility in some of these materials. The studies of the extended X-ray absorption fine structure (EXAFS) make it possible to estimate the lengths of bonds between emitting atoms and the nearest 
neighbours, the type of site occupancy in the lattice, and the degree of ordering by examination of the Debye-Waller factors [1]. EXAFS is then the unique technique giving the access to the local order in studied materials. This is especially important for the multi-component materials where the location of atoms in the crystal units cannot be indicated a priori, and the lengths of bonds can differ considerably from those resulting from the X-ray diffraction [2]. The presented investigations are part of systematic studies of short-range order in II-VI and IV-VI matrices with transition and rare earth metals.

\section{Experiment and results}

$\mathrm{Zn}_{1-x} \mathrm{Co}_{x} \mathrm{~S}$ and $\mathrm{Zn}_{1-x} \mathrm{Co}_{x}$ Se specimens were grown by chemical vapour transport using iodine as a transport agent. The chemical composition of the crystals was determined by electron probe microanalyses using a Jeol JSM-50A.
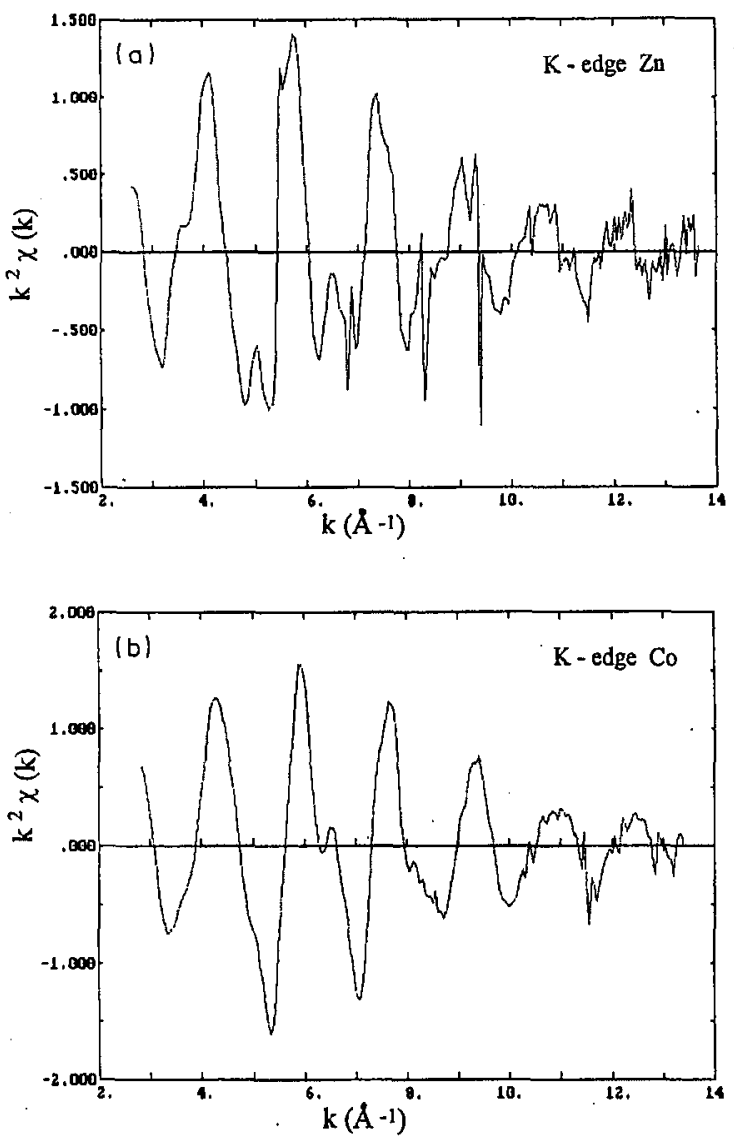

Fig. 1. Normalised EXAFS oscillations for (a) the Zn $K$-edge and (b) the Co $K$-edge of $\mathrm{Zn}_{1-x} \mathrm{Co}_{x} \mathrm{~S}, x=0.25$. Similar oscillations were observed for all composition range. 

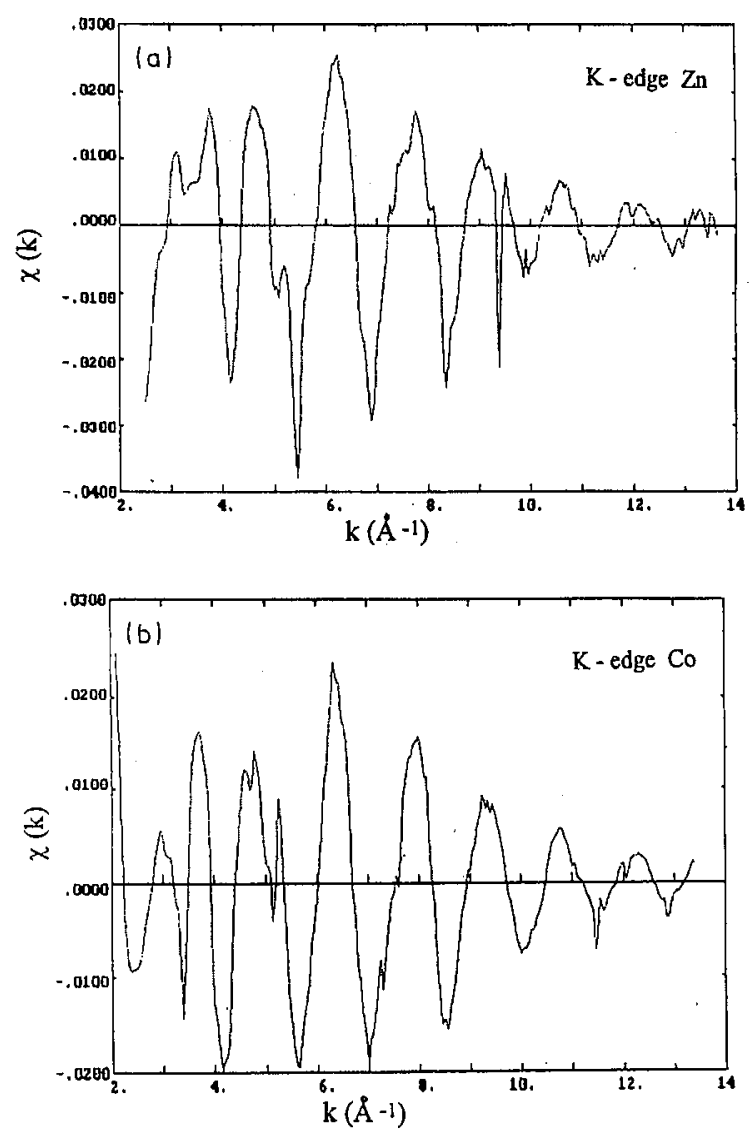

Fig. 2. Normalised EXAFS oscillations at (a) the Zn $K$-edge and (b) the Co $K$-edge of $\mathrm{Zn}_{1-x} \mathrm{Co}_{x} \mathrm{Se}, x=0.07$. Similar oscillations were observed for all composition range.

According to these measurements, the Co content of the investigated samples was $x=0.05,0.10,0.16,0.25$ for sulphides and $x=0.02,0.07$ for selenides. The $\mathrm{X}$-ray diffraction studies performed with a conventional X-ray powder diffractorreter ( $\mathrm{Cu} K_{\alpha}$ radiation) confirmed that all the sarmples reveal zinc blende structure. The diffractograms showed no trace of another phase.

The EXAFS measurements were performed at room temperature in transition mode at the XAS1 station on DCI ring at LURE, Université de Paris-Sud, France. The measurements included the $K$-edges of cation $\mathrm{Zn}$ and Co. The absorption spectra were analysed according to standard procedure. The EXAFS oscillations were obtained by subtraction of the pre-edge and atomic contribution. The pre-edge region was fitted with linear function. The absorption above the edge was fitted with splines to simulate the atomic cross-section. Normalised EXAFS oscillation for the $Z_{n} K$-edge and Co $K$-edge of $Z_{n_{1-x}} \mathrm{Co}_{x} \mathrm{~S}, x=0.25$ are shown in Fig. 1 and for $\mathrm{Zn}_{1-t z} \mathrm{Co}_{t z} \mathrm{Se}, \boldsymbol{x}=0.07$ in Fig. 2. Similar oscillations were observed for the investigated composition range. The datia were multiplied by $k^{2}$ and Fourier 

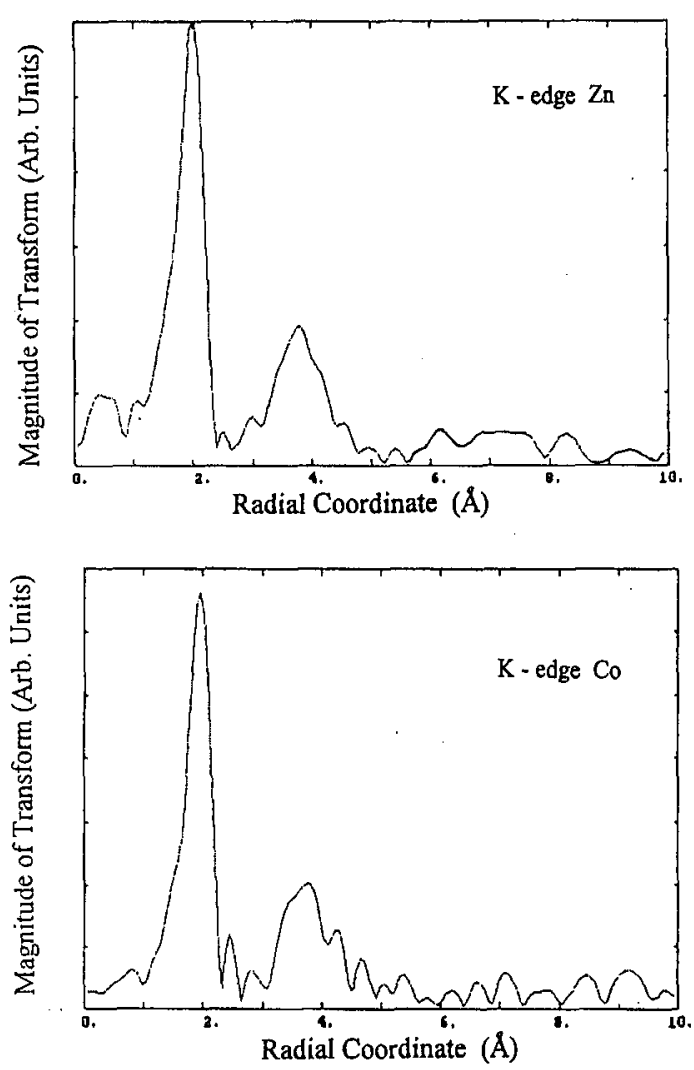

Fig. 3. Magnitude of Fourier transform of the $\mathrm{Zn} K$-edge and the Co $K$-edge of $\mathrm{Zn}_{1-x} \mathrm{Co}_{x} \mathrm{~S}$ for $x=0.16$. To separate the first shell oscillations the back Fourier transform in the range $0.86<R<2.7$ for $\mathrm{Zn}$ and $1.0<R<2.7$ for Co $K$-edge data was performed.

transformed in the range usually from $2.6-2.8$ to $13.4-14 \hat{A}^{-1}$ with Kaiser window in order to separate the contribution from various shells.

Magnitudes of Fourier transforms of the $\mathrm{Zn}$ and $\mathrm{Co} K$-edges for $\mathrm{Zn}_{1-x} \mathrm{Co}_{i x} \mathrm{~S}$ $(x=0.16)$ and for $\mathrm{Zn}_{1-x} \mathrm{Co}_{x} \mathrm{Se}(x=0.07)$ are shown in Fig. 3 and Fig. 4 , respectively. To separate the first shell oscillations the back Fourier transform in the range $0.86<R[\AA]<2.7$ for $Z$ n and $1.0<R[\hat{A}]<2.7$ for Co $K$-edge data in sulphides and in the range $1.3<R[\AA]<2.7$ for selenides was performed.

The structural parameters characterising the first coordination shell around cation can be obtained from an analysis of first shell EXAFS oscillations. The interatomic distances cation-anion $R$ and mean relative displacements $\sigma$ of each bond were estimated in our analysis. All investigated compounds have crystallised in zinc blende structure and each cation has four nearest neighbouring atoms. Therefore the coordination nurrber $N$ was treated as a known parameter equal to 4. The standard EXAFS formula [3] was used to simulate the first shell os- 

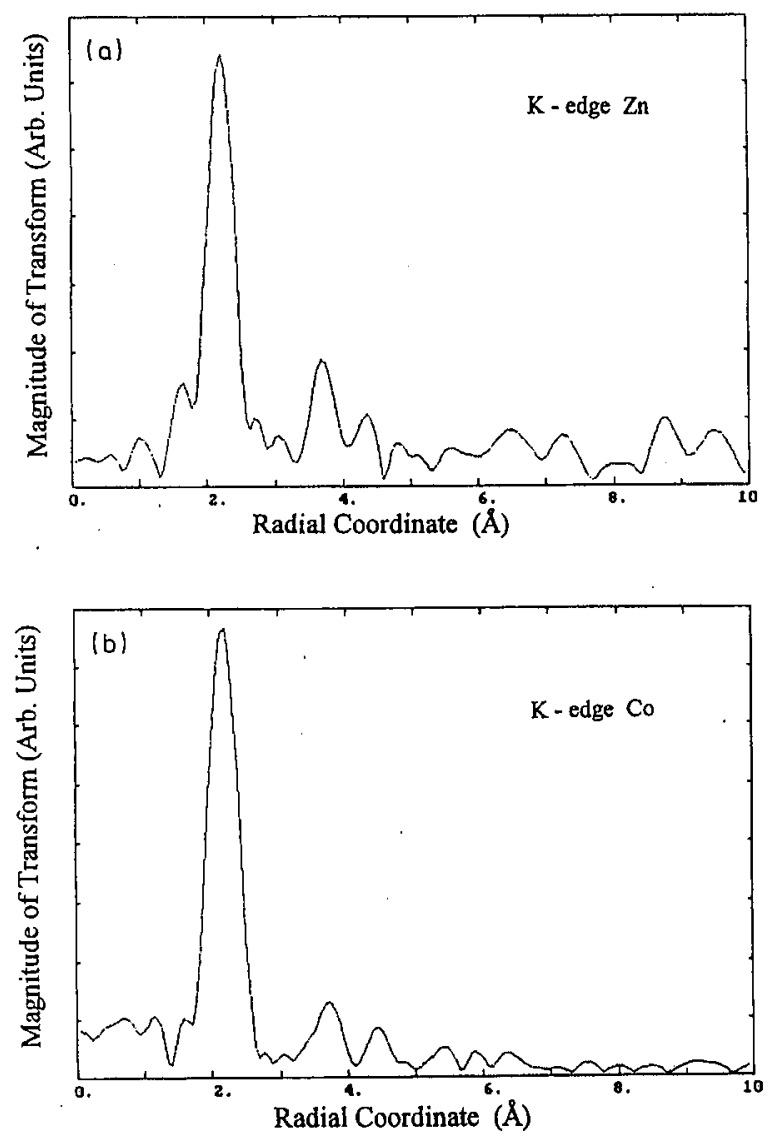

Fig. 4. Magnitude of Fourier transform for (a) the Zn $K$-edge and (b) the Co $K$-edge of $\mathrm{Zn}_{n_{1-x}} \mathrm{Co}_{x} \mathrm{Se}, x=0.07$. To separate the first shell oscillations the back Fourier transform in the range $1.3<R<2.7$ was performed.

cillation with $R$ and $\sigma$ left as free parameters. The theoretical phase shifts and amplitude were taken from McKale [4], the parameter $L$ is related to the mean free path of scattered photoelectron by expression $\exp (-2 R / L)$. The values of the best fit parameters are listed in Table I and Table II for sulphides and selenides, respectively. $\Delta E$ indicated a shift of the $K$-edge position $E_{0}$, the fit is a sum of differences at each point between the least squares fit of first shell EXAFS formula and experimental first shell oscillation.

Filtered $\mathrm{Zn}$ and Co $K$-edge $k^{2} \chi(k)$ data (solid line) and least squares fit (dotted line) for $\mathrm{Zn}_{1-x} \mathrm{Co}_{x} \mathrm{~S}, x=0.25$ and for $\mathrm{Zn}_{1-x} \mathrm{Co}_{x} \mathrm{Se} x=0.07$ are shown as examples in Fig. 5 and Fig. 6, respectively. The quality of fitting is similar for different contents of Co (see Table I and II). The coordination $N$ was then allowed to vary to check if there were many vacancies in investigated crystals and we found for sulphides with $x=0.10$ and 0.16 a better fit when $N$ was a little bit smaller 
TABLE I

Values of the structural parameters obtained from the best fits of the first shell EXAFS signal for $\mathrm{Zn}$-anion and Co-anion bonds in $\mathrm{ZnCoS}$. Theoretical phase and amplitudes used.

\begin{tabular}{c|c|c|c|c|c|c}
\hline \hline$K$-edge $\mathrm{Zn}$ & $\mathrm{R}_{\mathrm{Zn}-\mathrm{S}}[\hat{\AA}]$ & $\sigma[\hat{\AA}]$ & $L$ & $N$ & $\Delta E[\mathrm{eV}]$ & Fit \\
\hline $\mathrm{ZnS}, x=0.0$ & 2.344 & 0.072 & 5.83 & 4.00 & -7.86 & 0.083 \\
$\mathrm{ZnCoS}, x=0.05$ & 2.346 & 0.071 & 5.83 & 4.00 & -7.01 & 0.050 \\
$\mathrm{ZnCoS}, x=0.10$ & 2.344 & 0.074 & 5.83 & 4.00 & -6.99 & 0.071 \\
$\mathrm{ZnCoS}, x=0.16$ & 2.346 & 0.069 & 5.83 & 4.00 & -7.15 & 0.077 \\
$\mathrm{ZnCoS}, x=0.25$ & 2.344 & 0.068 & 5.83 & 4.00 & -7.15 & 0.163 \\
\hline$K$-edge Co & $R_{\mathrm{Co}-\mathrm{S}}[\hat{\AA}]$ & $\sigma[\hat{A}]$ & $L$ & $N$ & $\Delta E[\mathrm{eV}]$ & Fit \\
\hline $\mathrm{CoS}, x=0.00$ & 2.288 & 0.079 & 2.96 & 6.00 & -7.5 & 0.440 \\
$\mathrm{ZnCoS}, x=0.10$ & 2.322 & 0.063 & 5.83 & 4.00 & -5.72 & 0.194 \\
$\mathrm{ZnCoS}, x=0.16$ & 2.316 & 0.061 & 5.83 & 4.00 & -6.15 & 0.251 \\
$\mathrm{ZnCoS}, x=0.25$ & 2.316 & 0.058 & 5.83 & 4.00 & -6.20 & 0.113
\end{tabular}

TABLE II Values of the structural parameters obtained from the best fits of the first shell EXAFS signal for $\mathrm{Zn}$-anion and Co-anion bonds in $\mathrm{ZnCoSe}$. Theoretical phase and amplitudes used.

\begin{tabular}{c|c|c|c|c|c|c}
\hline$K$-edge $\mathrm{Zn}$ & $R_{\mathrm{Zn}-\mathrm{Se}}[\hat{\AA}]$ & $\sigma[\hat{\AA}]$ & $L$ & $N$ & $\Delta E[\mathrm{eV}]$ & Fit \\
\hline $\mathrm{ZnSe}, x=0.00$ & 2.456 & 0.078 & 5.83 & 4.00 & -2.03 & 0.356 \\
$\mathrm{ZnCoSe}, x=0.02$ & 2.453 & 0.075 & 5.83 & 4.00 & -3.86 & 0.196 \\
$\mathrm{ZnCoSe}, x=0.07$ & 2.455 & 0.073 & 5.83 & 4.00 & -5.11 & 0.126 \\
\hline$K$-edge Co & $R_{\mathrm{Co}-\mathrm{Se}}[\hat{\AA}]$ & $\sigma[\AA]$ & $L$ & $N$ & $\Delta E[\mathrm{eV}]$ & Fit \\
\hline CoSe, $x=0.00$ & 2.425 & 0.102 & 5.83 & 6.00 & 1.13 & 0.186 \\
$\mathrm{ZnCoSe}, x=0.07$ & 2.433 & 0.054 & 5.83 & 4.00 & -3.36 & 0.258
\end{tabular}

\section{TABLE III}

Values of the structural paraneters obtained from the best fits of the first shell EXAFS signal for Zn-anion bonds in $\mathrm{ZnCoS}$. Experimental phase and amplitudes from ZnS standard used.

\begin{tabular}{c|c|c|c|c|c}
\hline$K$-edge Zn & $R_{\mathrm{Zn}-\mathrm{S}}[\AA]$ & $\sigma[\AA]$ & $L$ & $\Delta E[\mathrm{eV}]$ & Fit \\
\hline $\mathrm{ZnS}, x=0.0$ & 2.344 & 0.072 & 5.83 & -7.86 & 0.083 \\
$\mathrm{ZnCoS}, x=0.05$ & 2.345 & 0.073 & 5.83 & 0.64 & 0.054 \\
$\mathrm{ZnCoS}, x=0.10$ & 2.350 & 0.075 & 5.83 & -0.7 & 0.068 \\
$\mathrm{ZnCoS}, x=0.16$ & 2.353 & 0.069 & 5.83 & -0.76 & 0.052 \\
$\mathrm{ZnCoS}, x=0.25$ & 2.349 & 0.068 & 5.83 & -0.92 & 0.148
\end{tabular}



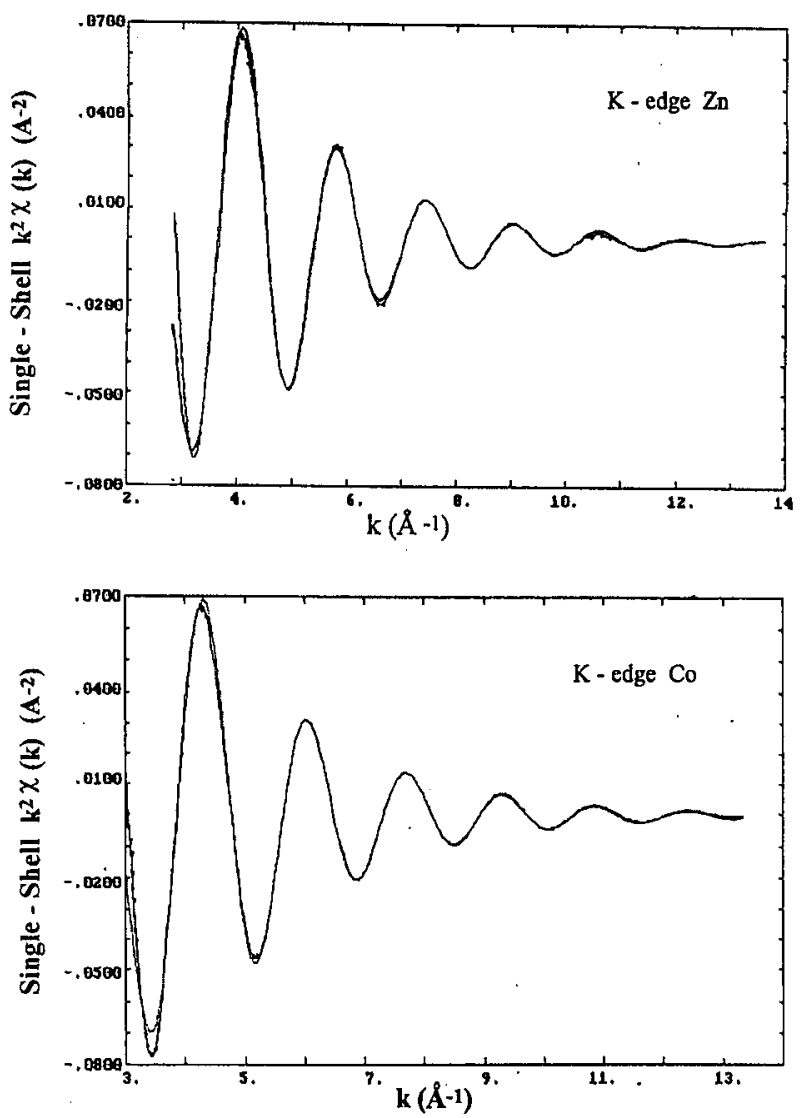

Fig. 5. Filtered $\mathrm{Zn}$ and Co $K$-edge $k^{2} \chi(k)$ data (solid line) and least squares fit (dotted line) for $\mathrm{Zn}_{1-x} \mathrm{Co}_{x} \mathrm{~S}, x=0.25$.

than 4. For all other crystals the change in coordination number does not improve the quality of fitting.

The value of the relative mean displacement $\sigma$ found in our fitting procedure is smaller than the Debye-Waller factor estimated from diffraction measurement

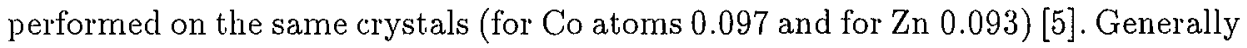
we observed a tendency for ordering with increase in Co content in investigated crystals and the $\sigma$ values were found to be smaller for ternary compound than for binary standard.

The application of the experimental phase shifts and amplitudes in EXAFS analysis is known to provide better results than the theoretical ones. The experimental values of the phase shifts and amplitudes for standard birary alloys can be used under the assumption of their transferability from standard to investigated corropounds. The advantages of the experimental values to the theoretical ones lie in the fact that many-electron effects are left out of the calculations, and as a result the overall amplitude can be difficult to simulate correctly. We try to perform 

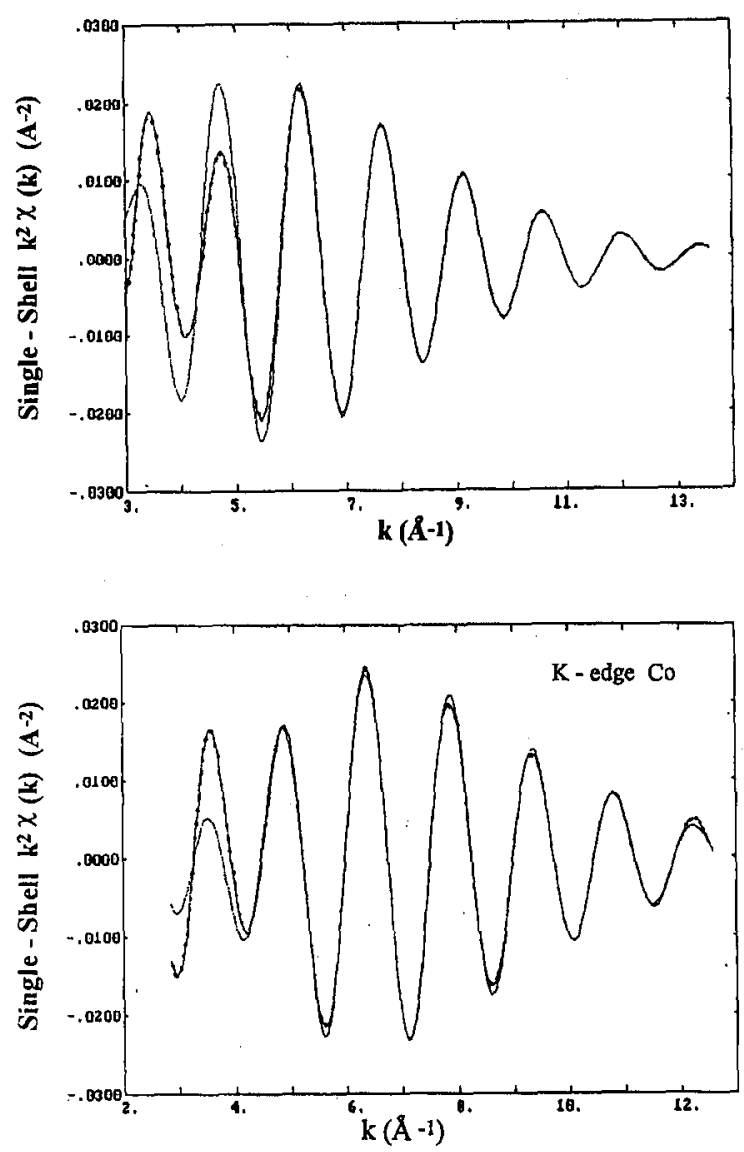

Fig. 6. Filtered $\mathrm{Zn}$ and Co $K$-edge $k^{2} \chi(k)$ data (solid line) and least squares fit (dotted line) for $\mathrm{Zn}_{1-x} \mathrm{Co}_{x} \mathrm{Se}, x=0.07$.

the EXAFS analysis using $\mathrm{ZnS}$ as a standard. The results of the best fitting are presented in Table III. The resulting lengths of $\mathrm{Zn}-\mathrm{S}$ bonds do not differ more than error from that obtained from theoretical values of phase shifts and amplitudes. The same was found for CoS, ZnSe and CoSe used as a standard.

\section{Conclusion}

The performed analysis of EXAFS oscillations in Co doped $\mathrm{ZnS}$ and $\mathrm{ZnSe}$ matrices shows that the cation-anion distances $\mathrm{Zn}-\mathrm{S}(\mathrm{Se})$ or $\mathrm{Co}-\mathrm{S}(\mathrm{Se})$ are systematically shorter in $\mathrm{ZnS}$ than in ZnSe matrix. This is in agreement with the fact that atomic radius of $S$ is smaller than Se. The $\mathrm{Zn}-\mathrm{S}$ distances were found to be $2.344(0.005) \AA$ in investigated range of Co content. The Co-S bond was $0.026 \AA$ shorter than $\mathrm{Zn}-\mathrm{S}$ thus introducing the asymmetry in unit cell and causing the decrease in the lattice constant with increase in Co content as was found in diffraction measurements [6]. The $\mathrm{Zn}-\mathrm{Se}$ distances were estimated to be 2.455 
(0.005) in the studied range of Co content and $0.022 \AA$ larger than the Co-Se ones thus indicating the reason for the decrease in the lattice constant in Co doped $\mathrm{ZnSe}$. This allowed us to estimate the covalent radius of Co in the studied matrices to be $1.20 \AA$ that means $0.025 \AA$ smaller than the $\mathrm{Zn}$ covalent radius [7]. Our findings confirmed the statement [7] that covalent radius is associated with the specific atom and is independent of the type of surrounding atorns. The behaviour of Co dopant does not depend on the matrix and in both investigated cases causes similar distortion of lattice and the same change of bond leggth independent on the type of anion. Analysing in the same way the behaviour of $\mathrm{Mn}$ dopant in $\mathrm{ZnSe}$ [8] and $\mathrm{ZnS}$ [9] matrices we found the difference in $\mathrm{Zn}$ and $\mathrm{Mn}$ covalent radii -0.09 which led to the $\mathrm{Mn}$ covalent radii $1.315(0.01) \AA$ in agreement with covalent tetrahedral radius of $\mathrm{Mn}$ (1.326-0.018 $\AA$ ) reported by Yoder-Short et al. [10]. The tetrahedral radius of $\mathrm{S}$ atoms resulting from our investigation is $1.116(0.01) \AA$ and tetrahedral radii of Se atoms is $1.225(0.01) \AA$ that means $\mathrm{Zn}$ and Se atomic tetrahedral radii are equal.

The analysis of the Debye-Waller factors in studied materials indicated a better ordering in the ternary compounds than in the binary standard ones in agreernent with rocking curves [7].

\section{Acknowledgments}

We thank Prof. A. Fontaine, Dr S. Pizzini and Dr A. Traverse for assistance in the experiment at XAS station on DCI ring at LURE, Orsay, France. We are also grateful to Dr D. Bonin for the prograrn used in our EXAFS analysis. This work was supported in part by the grant No. 223149102 of the State Committee for Scientific Research (Republic of Poland).

\section{References}

[1] U. Scheuer, B. Lengeler, Phys. Rev. B 44, 9883 (1991).

[2] A. Balzarotti, N. Motta, A. Kisiel, M. Zimnal-Starnawska, M.T. Czyżyk, Phys. Rev. $B$ 31, 7526 (1985).

[3] B.K. Teo, EXAFS: Basic Principles and Data Analysis, Inorganic Chemistry Concepts, Vol. 9, Springer-Verlag, Berlin 1986, p. 26.

[4] A.G. McKale, B.W. Veal, A.P. Paulikas, S.K. Chan, G.S. Knapp, J. Am. Chem. Soc. 110, 3763 (1988).

[5] W. Paszkowicz, J. Domagała, to be published.

[6] K. Eawniczak-Jabłońska, Z. Gołacki, W. Paszkowicz, J. Masek, L.-S. Johanson, M. Heinonen, J. Phys. Condens. Matter. 6, 3369 (1994).

[7] W. Giriat, J.K. Furdyna, in: Diluted Magnetic Semiconductors, Ed. J.K. Furdyna, J. Kossut, Academic Press, London 1988, p. 6.

[8] W.-F. Pong, R.A. Mayanovic, B.A. Bunker, J.K. Furdyna, U. Debska, Phys. Rev. $B 41,8440(1990)$.

[9] R. Iwanowski, K. Eawniczak-Jabłon'ska, to be published.

[10] D.R. Yoder-Sliort, U. Debska, J.K. Furdyna, J. Appl. Phys. 58, 4056 (1984). 